\title{
Obturator Nerve Block for Management of Chronic Pelvic Pain
}

\author{
Mercy A. Udoji MD
}

Every year, thousands of women undergo surgeries to treat severe female pelvic organ prolapse or stress urinary incontinence. Unfortunately, chronic pelvic pain may result from these surgical interventions, especially if mesh was used. This case report describes the management of 2 patients that presented at an outpatient pain center with chronic pelvic pain secondary to obturator neuralgia.

The first patient was a 45-year-old with history of vaginal reconstruction surgery, who presented to the pain clinic with severe pain in the medial thigh radiating to the perineal area. This pain had been present for months and persisted despite use of oral opioids, neuropathic pain medications, and topical agents. The patient had been seen and evaluated by neurology and urogynecology post operatively, but they could not ascertain the cause of her chronic complaints. The second patient was a 47-year-old with pain in the medial thigh and left side of her groin after transobturator sling procedure. Her pain was resistant to oral neuropathic pain medications (gabapentin and pregabalin) primarily due to the patient's inability to tolerate therapeutic doses of these medications. She was referred to neurology and nerve conduction studies was notable for decreased conduction in the left obturator nerve.

Both patients chose to try ultrasound guided obturator nerve blocks as a diagnostic and treatment modality. After the injection, the patients endorsed significant relief of their pain that persisted through their 3 month follow-up appointments leading to improved functionality in many aspects of their daily lives.

In a clinical situation like the one described above, the pain practitioner should more readily consider use of these blocks in the outpatient setting for pelvic pain patients whose symptoms are suggestive of obturator neuralgia.

Key words: Chronic pelvic pain, obturator nerve block, mesh pain, obturator neuralgia
Every year, more than 200,000 women undergo surgical procedures for management of symptoms related to stress urinary incontinence (SUI) or pelvic organ prolapse (POP). Unfortunately, surgical interventions aimed at treating these debilitating conditions can cause serious complications, especially chronic pelvic pain (CPP). CPP is described as a continuous or noncontinuous pain located in the lower abdomen or pelvis, which may be cyclical in nature and causes significant disruption and functional limitation for the

Author for correspondence: Mercy A. Udoji, MD, CMQ

Assistant Professor, Emory University

Department of Anesthesia

Staff Anesthesiologist, Atlanta VA Medical Center

Atlanta, GA

E-mail: mercy.udoji@va.gov affected patient (1). The etiology of CPP is multifactorial and may include disturbances in the urologic, gynecologic, gastrointestinal or neurologic systems. The incidence of CPP secondary to placement or removal of mesh during vaginal sling procedures is estimated to be as high as $30 \%$ (2). Pain in this scenario, is often secondary to pudendal nerve dysfunction, obturator nerve dysfunction, tissue damage, chronic inflammation, and other entities. We present the first case series describing use of ultrasound guided obturator nerve block for the management of CPP secondary to obturator nerve disruption.

\section{CASE REPORTS}

The local institutional review board was consulted and formal review was deemed unnecessary. 


\section{Case One}

The patient is 45-year-old woman with medical history notable for hypertension, asthma, and mitral valve prolapse, who presented to our outpatient clinic for evaluation of chronic, right sided pelvic pain. Her pain began approximately 6 months after vaginal reconstructive surgery (anterior and posterior colporrhaphy, extraperitoneal colpopexy via sacrospinous suspension) for POP. The patient had also undergone sacrospinous suspension 3 years previously for similar symptoms. This pain presented in the immediate post-operative period and was so severe that the patient required readmission on post-operative day 4 for pain control. Pain has persisted since her surgery despite use of oral opiates (acetaminophen and hydrocodone combination) and neuropathic pain medications (gabapentin and compounded pain cream). Post operatively, the patient had also been evaluated multiple times by neurology and urogynecology and continued not to improve. Magnetic resonance imaging (MRI) of the pelvis was notable for some tendinosis and tendonitis, but did not point to a specific etiology of patient's pain.

During her initial evaluation, the patient reported constant pain located in her medial thigh, radiating into her vagina and rectum. On physical examination, patient had some allodynia and hyperesthesia of upper medial thigh/groin region. This pain was described as debilitating, effectively preventing the patient from functioning at her preoperative levels and limiting her ability to perform activites of daily living and damaging patient's relationship with her spouse. Patient considered this pain debilitating stating that she had not been able to function near her preoperative level as a result. At the conclusion of her visit, the diagnosis of CPP secondary to obturator neuralgia was made. At that time, treatment options were discussed with the patient (including medication changes, pelvic physical therapy/manipulation, nerve blocks) and she chose to undergo an obturator nerve block.

After informed written consent was obtained, $0.25 \%$ bupivacaine and triamcinolone acetonide was injected under ultrasound guidance into the fascial plane between the obturator longus and brevis as well as obturator brevis and magnus muscles. Patient endorsed pain relief within 30 minutes of the procedure and was discharged home soon after. At her one month follow-up visit, the patient reported $60 \%$ decrease in pain intensity. She continued to endorse $50-60 \%$ relief of pain from the injection at the 2 month follow-up visit, however, still complains of pain in the rectum and posterior thigh.

\section{Case Two}

The patient is a 47-year-old woman with medical history notable for hypothyroidism, hyperlipidemia, mitral valve prolapse and stress urinary continence that presented to our outpatient pain clinic for evaluation of CPP. She had undergone a transobturator sling procedure complicated by mesh erosion and subsequent excision 2 years prior to our encounter. At that initial visit, the patient's primary complaints included chronic medial thigh pain, groin pain, and weakness in the left lower extremity. Nerve conduction studies were notable for decreased conduction in her left obturator nerve. She had tried pregabalin and gabapentin in the past, but was unable to tolerate these medications secondary to their side effects prompting her to seek alternative forms of management. Treatment options were discussed with the patient and she chose to try amitriptyline as well as an obturator nerve block. Informed written consent was then obtained and patient underwent ultrasound guided obturator nerve block with $0.25 \%$ bupivacaine and triamcinolone acetonide. Amitriptyline $10 \mathrm{mg}$ at bedtime was also added to the patient's medication regimen. At her 2 month follow-up visit, patient reported that the nerve block led to near $100 \%$ relief of her pain for approximately one month prior to gradual return of pain to pre-procedure levels. At that visit, she underwent a second nerve block using the same technique described above. After this injection, patient reported $>10$ weeks of near complete relief of her pain that persisted past her 3 month follow-up visit. In addition, the patient endorsed improved functional status, decreased pain frequency and severity, and improved ability to participate in physical therapy due to the procedures.

\section{DISCUSSION}

POP and SUI affects millions of women in the United States yearly. Their exact incidence is difficult to ascertain due to the variable measurements/ diagnostic criteria, but can be greater than $40 \%$ in 
some population studies $(3,4)$. Treatment options include surgery, pessaries and pelvic floor muscle training. In the past 2 to 3 decades, mesh surgery has become the primary surgical treatment for SUI and POP, bolstered by ease of adoption and encouraging short term follow up studies $(2,5)$. Over time, reports in national news, social media, as well as plaintiff's lawyer commercials have made it clear to patients and providers alike that mesh surgery can be fraught with complications mandating the risk and benefits of the procedure be carefully weighed prior to choosing this type of intervention. Cementing these concerns is the fact that the Food and Drug Administration (FDA) has issued 2 separate warnings concerning mesh use and their many complications (2008 and 2011) (5).

CPP may result from surgical management of CPP and SUI. When it occurs, CPP may be due to nerve entrapment, mesh contraction or retraction, inflammation, or scarring (4). There are case reports and retrospective studies indicating that removal of the mesh component or laparoscopic neurolysis can be utilized for successful management of these patients. However, these procedures are not risk free and carry no guarantees. As an example, in the retrospective study published by Lee et al (5), 58 women underwent surgical excision of mesh secondary to CPP and approximately $25 \%$ of them obtained symptomatic relief or improved function post procedure. The variable success rate in the published literature $(2,6)$ places the patient in the unenviable position of choosing between living in continual pain or pursing a costly treatment option that may not relieve their symptoms, highlighting the need for a minimally invasive management technique (5).

In 2007 Halayel et al (7) published the first case series describing use of ultrasound to perform obturator nerve blocks (ONBs) in patients undergoing anterior cruciate ligament repair. While there have been other similar reports scattered about the literature, ours is the first (to the best of our knowledge) to describe use of the ONB in management of CPP secondary to surgical management of SUI and POP.

The obturator nerve (composed of the ventral rami of the second through fourth lumbar nerves)

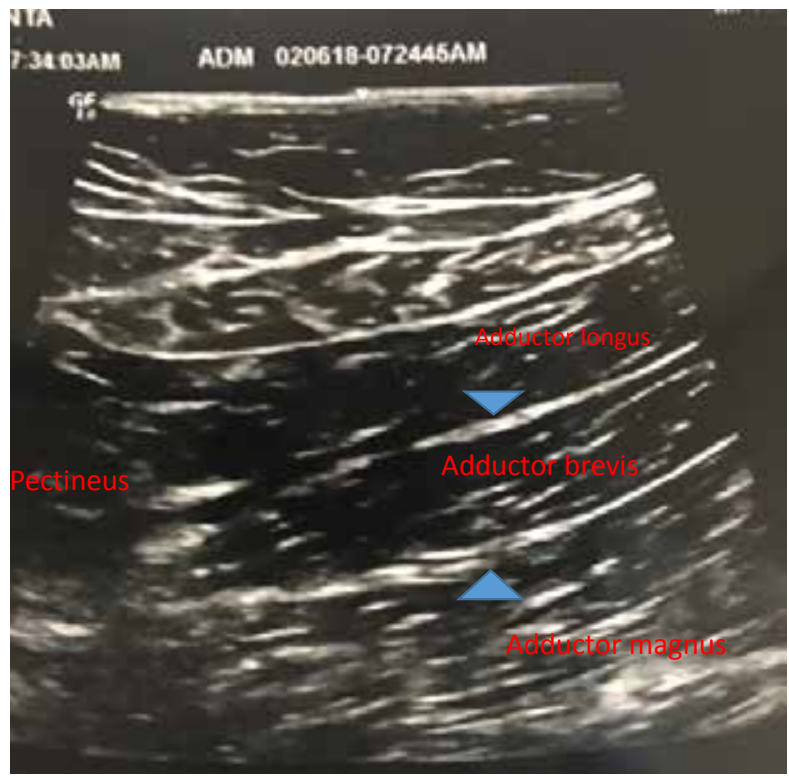

Fig. 1. Ultrasound view of the obturator nerve after it has divided into the anterior and posterior divisions. The anterior division (blue arrowhead pointing down) is found in the fascial plane between the adductor longus and brevis muscles. The posterior division (blue arrowhead pointing up) is found in the fascial plane between the adductor brevis and magnus muscles.

is formed in the psoas muscle and emerges from the medial border of the muscle at the pelvis (8). Branches of the obturator nerve innervate muscles of the medial compartment of the thigh including the adductor longus, gracilis, adductor brevis, and adductor magnus. Obturator neuropathy is characterized by continual pain in the upper medial thigh and groin area as well as sensory changes in the medial thigh and occasional muscle weakness. Disruption of the obturator nerve is rare and most often secondary to tumor compression, hemorrhage, or surgical insult (8).

The patients were placed in a supine position with the leg on the affected side slightly flexed and externally rotated at the hip. The inguinal ligament and pubic symphysis was identified by palpation. Then, a curvilinear (low frequency) ultrasound probe was used to identify the femoral nerve and artery, the sartorius, and the pectineus musculature. The probe was guided medially, at the junction of the pelvis and the lower extremity until 
the adductor longus, brevis, and magnus muscle group was identified medial to the pectineus muscle. The ultrasound probe was also utilized to identify the neurovascular bundle located between these muscle groups representing the anterior and posterior divisions of the obturator nerve (Fig. 1). Local anesthesia was achieved using $1 \%$ lidocaine. Then, a 22-gauge needle was guided using an out of plane technique through the skin and subcutaneous tissue first to the fascial plane between the adductor brevis and magnus and then to the fascial plane between the adductor longus and brevis and the injectate was divided between these 2 locations. The needle was removed and patient was monitored in the recovery room prior to discharge.

The nerve blocks described above were performed using the interfascial injection technique (after the division of the nerve into its anterior and posterior branches) described by Manassero et al (9) in 2012 due to ease of performance of the block and equivalent clinical efficacy. The same provider (MAU) performed all of the injections described. By targeting both anterior and posterior division of the nerves, we obviated the need to locate the nerve in the upper medial thigh and ensured that the entire nerve was blocked even in a situation where it divided high in the pelvis (approximately $50 \%$ of the time based on cadaveric studies) (10).

The successful reduction of our patients' pain symptoms indicates that ultrasound guided ONBs have a role in the management of chronic pain in the outpatient setting. Once a patient is deemed an appropriate candidate, the injection can be performed in a short amount of time in clinic, with minimal patient discomfort. Utilization of the ONB in this setting effectively expands the interventional pain practitioner's armamentarium, which may lead to decreased prescription of opiates and adjuvant pain medications as well as avoidance of their many adverse effects. Further, when pain is a complication of one procedure, a minimally invasive treatment option with direct, real-time visualization of structures may be preferred by patients as compared to a blind or stimulator driven technique that could cause more damage. Other patient-centered advantages include reduced cost, avoidance of more invasive procedures, minimal recovery time, and the relatively short time interval between block performance and the onset of analgesia.

\section{CONCLUSION}

In summary, the incidence of pelvic pain secondary to surgical management of POP and SUI is increasing. Ultrasound guided ONBs should be more frequently considered by interventional pain specialists as another tool to help manage this patient population. This technique is especially useful in patients that desire nonsurgical management of their condition or in whom medical management has resulted in intolerable side effects or inadequate pain control.

\section{REFERENCES}

1. Udoji MA, Ness TJ. New directions in the treatment of pelvic pain. Pain Manag 2013; 3:387-394.

2. Marcus-Braun N, Bourret A, Von Thoebald P. Persistent pelvic pain following transvaginal mesh surgery: A cause for mesh removal. Eur ] Obstet Gynecol Reprod Biol 2012; 162:224-228.

3. Kuncharapu I, Majeroni BA, Johnson DW. Pelvic organ prolapse. Am Fam Physician 2010; 81:1111-1117.

4. Hou JC, Alhalbi F, Lemack GE, Zimmern PE. Outcome of transvaginal mesh and tape removed for pain only. J Urol 2014; 192:856-860.

5. Lee D, Dillon B, Lemack G, Gomelsky A, Zimmern P. Transvaginal mesh kits-how 'serious' are the complications and are they reversible? Urology 2013; 81:43-48.

6. Corona R, De Cicco C, Schonman R, Verguts J, Ussia A,

Konickx PR. Tension-free vaginal tapes and pelvic nerve neuropathy. J Minim Invasive Gynecol 2008; 15:262-267.

7. Halayel PE, da Conceica DB, Pavei P, Knaesel JA, de Oliveira Filho GR. Ultrasound-guided obturator nerve block: A preliminary report of a case series. Reg Anesth Pain Med 2007; 32:221226.

8. Tipton JS. Obturator Neuropathy. Curr Rev Musculoskelet Med 2008; 1:234-237.

9. Manassero A, Bossolasco M, Ugues S, Palmisano S, De Bonis U, Coletta G. Ultrasound guided obturator nerve block. Interfascial injection versus a neurostimulation assisted technique. Reg Anesth Pain Med 2012; 37:67-71.

10. Soong J, Schafhalter-Zoppoth I, Gray AT. Sonographic imaging of the obturator nerve for regional block. Reg Anesth Pain Med 2007; 32:146-151. 